Revue internationale du CRIRES : innover dans la tradition de Vygotsky (2021), vol 5, no 1

\title{
Les théories historico-socioculturelles un intérêt nécessaire
}

\section{Sylvie Barma}

Professeure Titulaire

Comité d'Édition

Université Laval

Mélanie Gauthier

Comité de Coordination

melanie.gauthier.6@ulaval.ca

Doctorante en psychopédagogie

Université Laval

Qu'il s'agisse du partage de réflexions théoriques, méthodologiques, empiriques ou d'expériences professionnelles ancrées dans les milieux pratiques, la Revue internationale du CRIRES : innover dans la tradition de Vygotsky se veut un espace accessible à l'ensemble de la communauté des chercheurs, des praticiens et des étudiants des cycles supérieurs. Il s'agit dès lors d'un espace proposé pour et par la communauté des sciences de l'éducation, sociales et humaines plus que jamais nécessaire à l'exploration de ce monde en crise. Au fil des années, on remarque un intérêt grandissant pour les théories historico-socioculturelles. Ces théories s'inscrivent dans l'essence des travaux de Vygotsky et s'appuient sur l'idée selon laquelle les individus apprennent et se développent par la médiation individuelle et sociale et par l'utilisation d'artéfacts issus de la culture à laquelle ils appartiennent. À cela s'ajoutent les vecteurs d'influences liés à l'environnement à l'intérieur duquel l'individu évolue. «Qu'il apprenne, qu'il se forme, ou qu'il se développe, l'enfant, l'élève ou l'adulte ne constitue jamais une entité solitaire et décontextualisée : il agit sur-, réagit à-, se pose contre-, se met en lien avec-, etc. Bref, il interagit dans un environnement à la fois matériellement situé et historiquement déterminé » (Filliettaz, Schubauer-Leoni, 2008). En cette période atypique que nous traversons, il appert essentiel de remettre au monde un espace d'échange à haut rayonnement scientifique qui aborde des questions et des réflexions théoriques et pratiques en lien avec cette mouvance historique, sociale et culturelle.

Ce cinquième numéro, le premier d'une nouvelle mouture, s'inscrit dans le contexte très actuel que nous vivons. Un contexte lié simultanément aux transitions vécues en situation de crises dues aux imprévus (scolaires, familiaux, professionnels) et aux points de bifurcations auxquels le monde de l'éducation fait face (temps scolaire revisité, rôles à redéfinir, nouvelles formes d'alternance, nouvelles formes de médiations et de collaboration). Il débute par un texte l'école proposé par Stéphanie Duval, Marie Housen, Joanne Lehrer et Florence Pirard, intitulé : La pandémie, source de risques et d'opportunités pour la mise en place de pratiques de transition collaboratives lors de l'entrée à l'école qui nous présente un portrait des réalités reliées à la transition vers l'école en contexte de pandémie où la distanciation sociale et physique fait loi. Estce que ce contexte génère des différences dans la mise en place des pratiques d'accueil des jeunes 
élèves en rapport aux pratiques mises en place auparavant ? Est-ce que ce contexte de crise apporte avec lui la transformation des pratiques collaboratives qui normalement assurent la continuité dans les expériences vécues par les enfants ? Telles sont les questions que cet article ausculte tout en tenant compte du fait que le contexte de pandémie actuel peut devenir une source de possibles risques, mais aussi d'occasions pour la mise en place de pratiques de collaboration entre l'école et la famille.

Nathalie Panissal et Marie-Claude Bernard nous proposent un article intitulé La formation de la pensée critique revisitée par l'approche historico-culturelle qui a pour objectif de répondre à l'appel à la formation critique. Le texte y présente deux paradigmes. Le premier étant celui qui cherche à caractériser la pensée critique en y identifiant les processus cognitifs impliqués, le deuxième étant celui qui, sous une lecture historico-culturelle, propose une pensée critique revisitée. Conséquemment, cet article expose des propositions éducatives découlant de ces avenues et qui s'inscrivent dans une perspective orientée sur la formation d'une pensée critique socialement ancrée dans une capacité à appréhender la complexité du monde actuel.

Le texte Collaborations interprofessionnelles et travail collectif dans une classe mixte genevoise : un environnement capacitant d'inclusion scolaire éclaire par les théories de l'activité proposé par Solange Ciavaldini-Cartaut, Edith Guilley, Verena Jendoubi et Marion Dutrévis documente la façon dont s'introduit un environnement d'apprentissage et de travail propice à l'école inclusive dans un contexte situé. Les résultats que présente l'article témoignent de l'opérationnalisation du travail collectif dans une classe intégrée mixte (CLIM) du secondaire ainsi que des contradictions de premier niveau rencontrées. Cet article illustre qu'il est possible d'atteindre des objectifs d'inclusion scolaire chez les élèves en dépassant certaines contraintes inscrites dans le système d'activité.

Cette nouvelle édition se termine avec un texte de Maria Antonieta Impedovo intitulé Développement professionnel agentif et collaboratif des enseignants : communautés épistémiques via les réseaux sociaux en temps de crise. Sous le thème de l'agentivité, ce texte explore les perspectives liées au développement professionnel des enseignants par le biais des réseaux sociaux. L'autrice fait valoir les potentialités du réseautage comme clé des compétences professionnelles au sein des carrières enseignantes. Dans le contexte de crise actuelle, l'observation des groupes de réseaux sociaux liés au Covid-19 met en relief la façon dont les interactions des enseignants via internet peut faciliter certaines approches collaboratives en soutenant, notamment, l'engagement. Les réseaux sociaux étant perçus comme de tiers espaces entre l'apprentissage formel et informel le texte suggère qu'ils peuvent agir en soutien au développement professionnel.

C'est au nom du comité de rédaction et de coordination de ce nouveau numéro que nous partageons l'enthousiasme de la concrétisation d'une nouvelle ère quant à la publication et l'indexation de la Revue internationale du CRIRES: innover dans la tradition de Vygotsky. Nous souhaitons vivement que cet espace d'échange suscite l'intérêt de nombreux collaborateurs, chercheurs et professionnels, qui ont à coeur la compréhension et l'innovation des sciences de l'éducation, des réflexions et des pratiques éducatives soutenues par une tradition (et une transition) historique, sociale et culturelle au potentiel qui ne se dément pas. 